\title{
Active Sites and Ice Crystal Nucleation
}

\author{
N. H. FLETCHER \\ Dept. of Physics, University of New England, Armidale, N.S.W., Australia \\ (Manuscript received 29 April 1969, in revised form 13 August 1969)
}

\begin{abstract}
The concept of active nucleation sites is examined in the light of standard nucleation theory and it is suggested that these sites are probaly small re-entrant corners or jogs in growth steps on the surface of the nucleating particle. The nucleation behavior of a spherical particle with a conical pit on its surface is examined in detail and the activity of a population of particles having a log-normal distribution of such pits is worked out. The parameters involved in the theory can be evaluated within acceptable limits and the resulting curves exhibit the phenomena observed experimentally. Generalized activity curves are given in parametric form for more detailed comparison with experiment.
\end{abstract}

\section{Introduction}

Some time ago the present author (Fletcher 1959a, b) put forward a theoretical description of the nucleation behavior of aerosols, such as silver iodide smoke, which attributed their activity spectrum to their particle size distribution and the nucleation-inhibiting effects of surface curvature. The theory as presented made several assumptions about the nature and activity of the smoke produced by burning complex solutions of AgI-KI which have since proved incorrect, but a revised version (Fletcher, 1968), taking proper account of the effect of the soluble component, still makes the same basic assumption about the origin of the activity spectrum.

Reduced to its essentials, this assumption is that, to a sufficient approximation, aeŕosol particles of a welldefined material like AgI all have the same surface properties, characterized by a single parameter $m$, and differ only in size and perhaps shape. An opposite view has been taken by Katz (1962), by Edwards et al. (1962) and, more recently, by Mossop and Jayaweera (1969) who maintain, on the basis of experimental evidence, that there is a very wide range of nucleation activity even among the particles of a very nearly monodisperse preparation of AgI particles. They therefore attribute a major part of the activity spectrum to the distribution of "active sites" upon the surfaces of the nucleating particles.

It is the aim of the present paper to try to reconcile these two viewpoints and to examine the possible nature of these active sites.

\section{Nature of active sites}

The process of ice nucleation, whether it occurs through sublimination or freezing, has as its ratedetermining step the formation of an embryo ice crystal which is large enough to become free-growing in the surrounding supersaturated or supercooled environment. The details are set out in standard texts on cloud physics (for example, see Fletcher, 1962). A foreign particle promotes nucleation by providing a stable surface on which such an embryo can grow and so reduces the free energy penalty involved in the formation of a critical embryo. To be an efficient nucleus, the particle must be at least comparable in size with the critical embryo, and the free energy associated with the particle-ice interface must be as small as possible.

Several things may influence this interfacial free energy. The chemical nature of the particle is clearly important, since it influences the energy binding the water molecules in contact with its surface; so too is the crystallography of the exposed particle surfaces, since by binding water molecules in preferred configurations their ability to bond to each other is affected. These are, however, factors which enter equally for all similarly shaped particles of a given material. Rather more subtle is the effect of the distribution of ions on the particle surface, self-ions like $\mathrm{Ag}^{+}$or I- in $\mathrm{AgI}$ being the simplest case. This has been studied experimentally by Edwards and Evans (1962) following some theoretical suggestions by the present author (Fletcher, 1959c) and appears to be of considerable importance, greatest nucleation efficiency occurring near the isoelectric point. Again, however, one would expect the effect to be similar for all particles in a given aerosol.

To find a suitable model for an active site we must therefore look for some feature of smaller scale, and here nucleation theory can help us. The size of a critical embryo at a given temperature is well determined and, as we have already seen, the contact area between embryo and nucleating particle must be comparable with the total surface area of the embryo if the nucleation is to be efficient. Because it is the free energy of this interface which is important, the feature composing the 
active site must therefore be comparable in size with a critical embryo. It might be thought that a particularly low energy site of very small size might suffice but it is easily seen that this would simply capture a few water molecules, even in an unsaturated enviornment, and thenceforth behave simply like a small patch with $m=1$. To put some numerical values on this criterion, the diameter of a site active by freezing at $-\Delta T\left[{ }^{\circ} \mathrm{C}\right]$ is of order $200 / \Delta T[\AA]$ and, by sublimation, about $1000 / \Delta T[\AA]$, as follows from the usual discussion of the Kelvin equation.

Two possibilities then arise for the active sites. Either they represent the natural activity of bare substrate with the remainder of the surface covered by varying degrees of contamination, or else they are caused by some unusual surface feature of appropriate size. Zettlemoyer et al. (1963) performed experiments with hydrophobic material which suggested that impurities may play an important role in some cases, though in the direction of enhancing rather than inhibiting activity: Federer (1968) similarly ascribed variations in nucleation efficiency of semiconductor surfaces to the influence of impurities. It seems clear, however, that even very pure materials show a similar variability and we shall here restrict our attention to such cases.

The most likely form of surface variation is a smallscale topographical one. Such features are obviously capable of endless variation, are genuinely homogeneous to the material itself, and have the important property of gradually disappearing by surface diffusion, which accords with the fact that freshly produced particles are generally much more active than are well-aged particles.

The influence of surface topography on nucleation behavior was recognized by Turnbull (1950), who showed that a re-entrant cavity could greatly enhance nucleation efficiency and lead to retention of an embryo in unsaturated conditions. Several more general cases were later investigated by the present author (Fletcher, 1960). The general form of the conclusions is that, if the free energy barrier ${ }^{1}$ to homogeneous nucleation is $\Delta G_{0}{ }^{*}$, then the barrier for nucleation upon a plane surface of parameter $m$ is of the form

$$
\Delta G_{1}^{*}=\Delta G_{0}^{*}[(1-m) / 2] \text {. }
$$

For nucleation upon a step, the barrier is

$$
\Delta G_{2}^{*}=\Delta G_{0}^{*}[(1-m) / 2]^{2},
$$

and at a re-entrant step corner

$$
\Delta G_{3}^{*}=\Delta G_{0} *[(1-m) / 2]^{3},
$$

1 Abraham (1968) has advanced convincing arguments that the nucleation problem should be treated in terms of the Helmholtz free energy $F$, rather than the Gibbs free energy $G$. This does not affect the results in the present case and we use $G$ for consistency with our earlier notation.

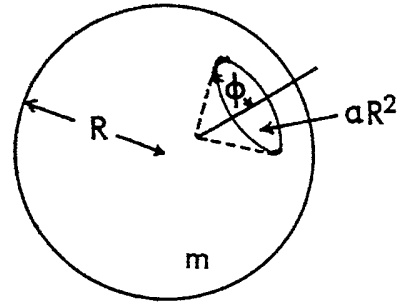

FIG. 1. Model for a nucleating particle as a sphere of radius $R$ and surface parameter $m$ containing a conical pit of semi-angle $\phi$ and surface area $\alpha R^{2}$.

where $m$ is defined by

$m=\left(\sigma_{\text {substrate/vapor }}-\sigma_{\text {substrate/embryo }}\right) / \sigma_{\text {embryo/vapor }}$,

$\sigma_{i j}$ being surface free energies. The value of $m$ lies between -1 and 1 so that the $\Delta G^{*}$ values given by (1)-(3) may be very much less than $\Delta G_{0}{ }^{*}$. These formulae are actually those appropriate for cubic crystals; corresponding relations for hexagonal geometry are similar, though rather more involved. In any case, the results only apply if the step or corner in question is large enough to accommodate a critical embryo; smaller topographical features have less effect.

It is clear that features of this kind are well able to account for the observed behavior; thus, suppose that the freezing threshold observed for AgI near $-4 \mathrm{C}$ is not characteristic of flat surfaces (requiring $m \approx 0.9$ ) but rather of the small number of particles having re-entrant corners of sufficiently large size. On a spherical approximation this only requires $m \approx 0.5$ and leads to a nucleation threshold for particles with simple steps at about $-10 \mathrm{C}$ and for featureless particles at about $-20 \mathrm{C}$. The height of step required for re-entrant corner nucleation at $-4 \mathrm{C}$ is about $50 \AA$, which is easily accommodated on the particles of radius $1000 \AA$ typically active at this temperature.

The purpose of this paper will be to explore the implications for nucleation phenomena of the existence of such topographical features upon the surfaces of nucleating particles. To do this we shall set up the simplest possible model capable of approximating reality and then see what its predictions are.

\section{Single-particle model}

Since we are seeking a model with the smallest possible number of parameters, we first accept that it is reasonable to neglect the crystalline form of both the nucleating particle and the growing ice embryo and to replace them by a sphere and spherical cap, respectively. The effect of this approximation has been studied in detail (Fletcher, 1963) and is seen not to be significant for the present study.

As a second approximation we shall idealize the crystalline nature of a re-entrant corner step and replace it by a re-entrant conical cavity of semi-angle $\phi$. A model nucleating particle containing such a feature then appears as shown in Fig. 1. It is characterized by its 
radius $R$ and the area of the conical pit on the surface, which we shall take to be $\alpha R^{2}$. The cone angle $\phi$ is presumed to be characteristic of the material of the particle, as also is the surface parameter $m$ which, in this approximation, is simply the cosine of the contact angle of ice upon the substrate material.

To investigate the nucleation behavior of such a particle we must first study the nucleation event in the conical cavity. This need not, however, concern us in detail. At some supercooling or supersaturation, whose value is determined by the angle $\phi$ and the surface parameter $m$ through a relation analogous to (3), nucleation of ice occurs in the vertex of the cavity and this ice crystal grows to fill the cavity completely, as in the analysis of Turnbull (1950). Above this threshold then, which we may take to be $-4 \mathrm{C}$ for AgI, no nucleation can occur, while below it the conical cavity can be considered to be filled with ice. The surface curvature of this ice insert is determined by the supercooling according to the usual Kelvin relation but, for our present purposes, we will make a negligible error by taking it equal to the curvature $R^{-1}$ of the host particle.

Our nucleation problem is now greatly simplified, since the nucleating particle can be taken to consist of a sphere of radius $R$ and surface parameter $m$, on the surface of which is a circular patch of area $\alpha R^{2}$ in which the parameter $m$ is unity. The nucleation behavior of such a particle can be treated in a straightforward manner by a slight extension of the approach for simple spherical particles. The resulting nucleation rate is

where

$$
J \approx 4 \pi R^{2} K \exp \left(-\Delta G^{*} / \dot{k} T\right),
$$

$$
\Delta G^{*}=\Delta G_{0}^{*} \cdot f(m, x)-\alpha R^{2}(1-m) \sigma .
$$

Here $\Delta G_{0}{ }^{*}$ represents the usual expression for the free energy barrier to homogeneous nucleation, $\sigma$ is the free energy per unit area of the ice-water interface $\left(\sigma \approx 20\right.$ ergs $\left.\mathrm{cm}^{-2}\right), x=R / r^{*}$ where $r^{*}$ is the radius of the critical embryo, and the function $f(m, x)$ is given by

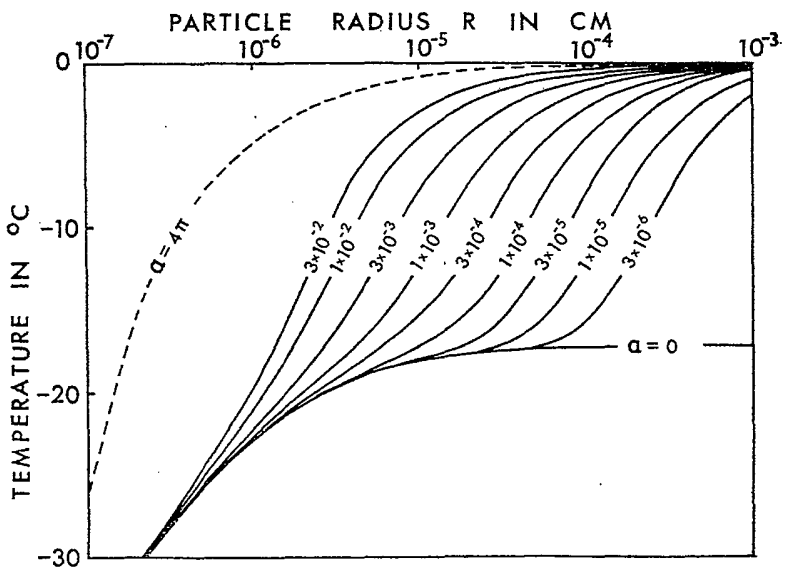

FIG. 2. Freezing nucleation behavior of a particle with surface parameter $m=0.5$ and having an active site of area $\alpha R^{2}$.
Fletcher (1963) [or, with a difference in definition of a factor 2, by Fletcher (1958)].

The kinetic coefficient $K$ in (5) depends upon details of the nucleation situation. For the case of freezing, the basic kinetic coefficient for homogeneous nucleation has been evaluated by Turnbull and Fisher (1949) and, multiplying by a factor of order $10^{-8}$ to convert from this volume problem to the surface problem involved in heterogeneous nucleation, we find $K \approx 10^{20} \mathrm{~cm}^{-2} \mathrm{sec}^{-1}$. Note that this corrected value is smaller than that used by the present author in earlier calculations by a factor of about $10^{6}$, the previous value being incorrect. As in most nucleation problems, this is not of great physical significance and simply leads to slightly larger values of the parameter $m$ being required.

The nucleation problem defined by (5) and (6) can now be solved by the usual methods (Fletcher, 1958) and yields the curves shown in Fig. 2 which give the temperature below which a particle characterized by a set of values of $R, m$ and $\alpha$ and in suspension in water will nucleate an ice crystal by freezing in less than $1 \mathrm{sec}$. These curves are drawn for the case $m=0.5$; curves for other values of $m$ are similar but are compressed or expanded in temperature range. It is clear that the presence of quite a small cavity has a very marked effect upon the nucleation behavior of particles $\gtrsim 100 \AA$ in radius, while for smaller particles the effect becomes negligible.

One addition should really be made to these curves before they are used in our further development. This is simply the imposition of an upper nucleation threshold corresponding to the supercooling required to nucleate an ice embryo within the cavity, which is reflected simply as a choice of the cavity angle $\phi$. However, as we shall see later, very few particles have a nucleation threshold as high as $-4 \mathrm{C}$, so that this refinement can safely be neglected.

It is perhaps appropriate to remark that exactly the same sort of general behavior is found for the case of growth of a simple cubic embryo upon a cubic nucleating particle on which is located a re-entrant cavity bounded by plane orthogonal faces. This case is reasonably easy to deal with from an algebraic point of view but is no more approximate to the case of ice than is the present idealization. It does, however, show automatically the threshold mentioned in the previous paragraph. A similar treatment using hexagonal geometry is quite feasible but introduces several complications because of the different configurations possible.

\section{Populations of particles}

In a real aerosol or aqueous suspension it is evident that each particle is different, but there is little to be gained from any theory which tries to include this diversity directly. Rather, we must seek a statistical description involving the minimum number of parame- 
ters necessary to describe the relevant properties. The effects of particle shape and of particle size have been treated in earlier discussions. We turn now to the parameters describing the distribution of surface pits or active sites.

One important observation is of great assistance here and that is the finding of Mossop and Jayaweera (1969) that, for the AgI particles which they studied, the probability that a particular particle possesses a site active at $-11 \mathrm{C}$ is proportional to its surface area. A similar conclusion was earlier reached by Edwards et al. (1962). It is reasonable to assume such findings to hold for all sites and to write the number of sites, per unit area, having active area in the range $A$ to $A+d A$, as $n(A) d A$. This is, indeed, the natural assumption to make, and any other would require special justification.

An additional assumption is required to establish the functional form of $n(A)$. Presumably, small cavities are more numerous than large ones and a variety of possible distributions might plausibly. be suggested. We are interested here in establishing the simplest possible theory with a minimum number of parameters, and the most reasonable assumption seems to be that either 1) the probability of a particle or radius $R$ possessing a pit of area $\alpha R^{2}$ is independent of $R$ and depends only on $\alpha$; or 2 ) the distribution of pit areas is of log-normal form.

The first assumption leads to a functional form

$$
n(A)=C / A \text {, }
$$

where $C$ is a constant. This distribution, however, gives an unreasonably large fraction of large pits and leads to an activity spectrum at variance with experiment. We therefore adopt the second alternative, noting that it is consistent with a model in which the pit sites are really jogs in growth steps on the surface, the steps themselves having a log-normal distribution of heights. The assumed functional form is thus

$$
n(A)=B \exp \left\{-\gamma^{2}\left[\ln \left(A / A_{0}\right)\right]^{2}\right\} .
$$

This distribution contains three parameters, $A_{0}, B$ and $\gamma$, which is unacceptably many, but we shall be able to assign reasonable limits to some of these and thus effectively reduce their number. To do this we note that a unit step on a crystal surface is one lattice parameter in height and that higher steps are generally formed by coalescence of these elementary steps. Taking a representative unit step as $5 \AA$, we may therefore reasonably assume for the parameter $A_{0}$ (which specifies the most probable pit size as due to the crossing of two unit steps) the value

$$
A_{0} \approx 2 \times 10^{-15} \mathrm{~cm}^{2},
$$

and limit the distribution (8) to $A \geq A_{0}$. The value of this parameter is, in any case, not critical. The extent to which coalescence of steps has proceeded will be characteristic of the particles concerned but one might expect even.double steps to be significantly less num- erous then single steps. For this to be the case, we must have for the parameter $\gamma$ (which specifies the width of the log-normal distribution) the value

$$
\gamma \approx 1 \text {. }
$$

Finally, we have the parameter $B$ which, with $A_{0}$ and $\gamma$ fixed, now measures the total roughness of the nucleating surface. From (8) the total fraction of surface area occupied by pits is

$$
\begin{aligned}
& \int_{A_{0}}^{\infty} n(A) A d A=B A_{0}^{2}(\sqrt{\pi} / 2) \exp \left(1 / \gamma^{2}\right) \\
& \times\left[1+\operatorname{erf}\left(1 / \gamma^{2}\right)\right] \approx 2 B A_{0}^{2}
\end{aligned}
$$

from which we conclude that

$$
B \ll A_{0}^{-2} \text {. }
$$

With the limitations expressed by (9), (10) and (12), we have now satisfactorily reduced the parameters involved in the distribution and can characterize the properties of different populations of particles primarily by a roughness parameter $\beta$ which we will define by

$$
\beta=B A_{0}^{2},
$$

which measures, essentially, the fraction of surface covered by pits. We must therefore have $\beta \ll 1$.

The operationally important quantity for nucleation theory is the probability that a particle of radius $R$ have on its surface at least one active site of area greater than $\alpha R^{2}$. The number of such pits per unit area is found by integrating $n(A)$ from $A=\alpha R^{2}$ to $A=\infty$ and is

$$
N\left(\alpha R^{2}\right)=\left(\beta / A_{0}\right)(\sqrt{\pi} / 2 \gamma) \exp \left(1 / 2 \gamma^{2}\right) \operatorname{erfc} x
$$

where

$$
x=\gamma \ln \left(\alpha R^{2} / A_{0}\right)-(1 / 2 \gamma) .
$$

Using standard approximations (Abramowitz and Stegun, 1965), this can be expressed in a form suitable for computation as

$$
N\left(\alpha R^{2}\right) \approx \frac{\beta}{\gamma A_{0}}\left[x+\left(x^{2}+1.5\right)^{\frac{1}{2}}\right]^{-1} \exp \left(\frac{1}{4 \gamma^{2}}-x^{2}\right) .
$$

By elementary statistical arguments (the Poisson distribution), the required probability is then

$$
P(\alpha, R)=1-\exp \left[-4 \pi R^{2} N\left(\alpha R^{2}\right)\right]
$$

which can be evaluated explicitly using (15) and (16).

\section{Application to silver iodide}

As the main object of this paper, let us apply the analysis given above to the case of silver iodide dispersions. We note the following experimental information.

(i) The threshold temperature for nucleation of ice formation by freezing is $-4 \mathrm{C}$ for large AgI particles. 
(ii) Not all large particles are active at this temperature and in fact we must lower the temperature to $-22 \mathrm{C}$ or less to achieve $100 \%$ activity for particles $\geq 100 \AA$ in radius (Mossop and Jayaweera, 1969).

(iii) The size distribution of typical AgI dispersions produced by smoke generators is closely log-normal. A median radius of $420 \AA$ and standard deviation factor 1.47 was found by Mossop and Tuck-Lee (1968) for the smoke they studied.

(iv) The smoke produced by acetone-solution burners does not consist of pure AgI particles but of hygroscopic mixed particles of AgI and NaI (Mossop and Tuck-Lee, 1968).

The information contained in (i) has previously been interpreted as implying a surface parameter $m \approx 0.9$ for AgI, but this is inconsistent with (ii). Instead, from (ii) we must now assume $m \approx 0.5$ and attribute the activity of a few particles at $-4 \mathrm{C}$ as due to re-entrant cavities. Indeed, a large cavity bounded by plane walls having $m=0.5$ and meeting at an angle of about $60^{\circ}\left(\phi=30^{\circ}\right)$ produces approximately the required threshold and cavities having this geometry are likely to occur in AgI.

Experimental point (iii), is straightforward -and a discussion of (iv) has been given in an earlier publica-

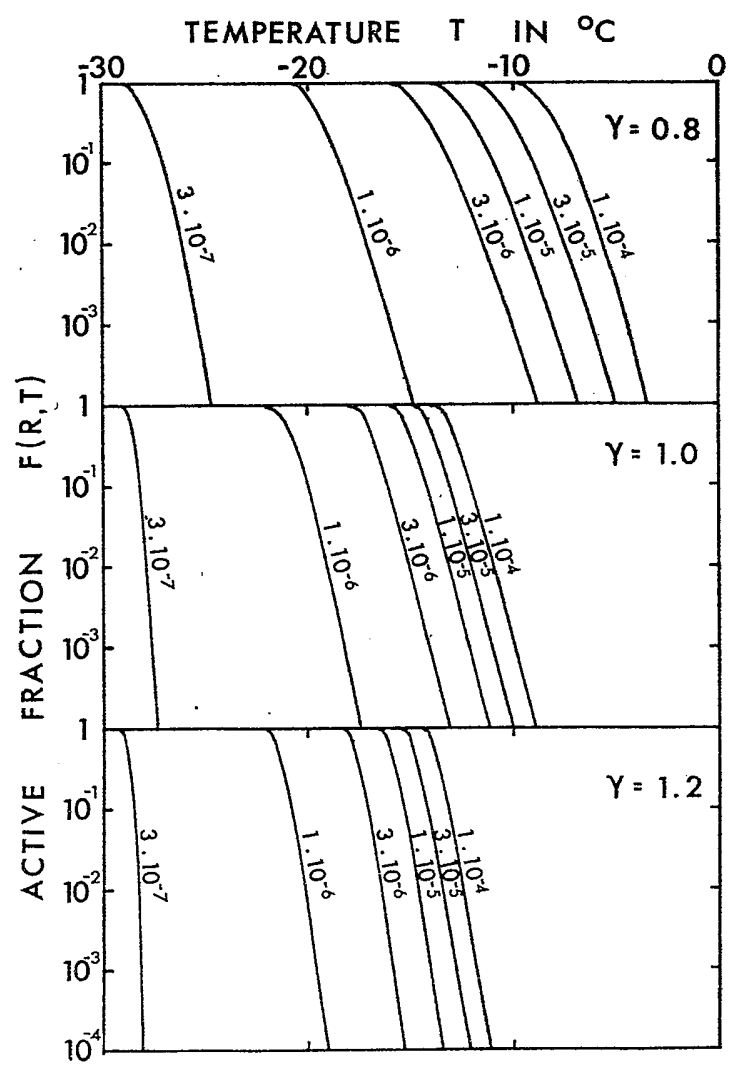

FIG. 3. Fractional activity $F(R, T)$ for freezing nucleation by a population of particles of radius $R$ and described by the parameters $\beta$ and $\gamma . R$ is shown as a parameter on each curve and in all cases $\beta$ is taken as 0.001 . tion (Fletcher, 1968). We shall not be concerned with these in the present paper.

Consider now an ensemble of particles of radius $R$ with a probability distribution $P(\alpha, R)$ of active sites upon their surfaces, as described by (17). With the value of $m$ assumed above, we have only to choose the parameters $\beta$ and $\gamma$ in accord with the relations $\gamma \approx 1, \beta \ll 1$ and it is then simple, with the aid of Fig. 2 , to calculate the activity distribution of the ensemble. If we define $F(R, T)$ to be the fraction of particles of radius $R$ active in nucleation above temperature $T$, then the results are as shown in Fig. 3. Here we have chosen $\beta=1 \times 10^{-3}$, which is physically reasonable, and the curves are plotted for three values of $\gamma$ close to unity.

The curve for a given particle size $R$ consists essentially of two straight lines: 1) a horizontal line at $F(R, T)=1.0$ below a critical temperature which depends upon $R$, and 2) a steeply sloping line above this temperature, the slope being of the order of a factor of 10 per degree with this value depending upon $\gamma$. From the form of Eqs. (16) and (17) it can be seen that the effect of a different choice of $\beta$ is essentially just to move the sloping part of the characteristic up or down by the same factor (the scale being logarithmic) and hence to change the point of its intersection with the line $F(R, T)=1$.

\section{Discussion}

It is immediately clear from these curves that the theory reproduces, to a good approximation, many of the effects observed experimentally. Large particles are more efficient nuclei than are small particles but for any given particle size there is a wide spread of nucleation thresholds. It is useful to display the results shown in Fig. 3 in a different way by plotting the temperature below which a given fraction $F$ of particles of radius $R$ act as freezing nuclei. This has been done in Fig. 4, still with the choice $\beta=10^{-3}$ and for two values of $\gamma$.

The curves in Fig. 4 are immediately reminiscent of the nucleation efficiency plots of the usual simple theory (Fletcher, 1958), although the shapes are somewhat different in detail. If the behavior of only a small fraction of particles is being considered, then they appear to have a larger value of the contact parameter $m$ than does the population as a whole. In other words, the particle population behaves as if it possesses a distribution of $m$ values from $m=0.5$, corresponding to a threshold of $-20 \mathrm{C}$ for large particles, up to $m=0.9$, corresponding to a threshold of $-4 \mathrm{C}$ for a very small fraction of particles. (In the simplified form of the theory presented here the $m$ distribution actually extends to $m=1.0$, giving a threshold of $0 \mathrm{C}$, but we have discussed in an earlier section the reason why this should be truncated at $-4 \mathrm{C}$.) 


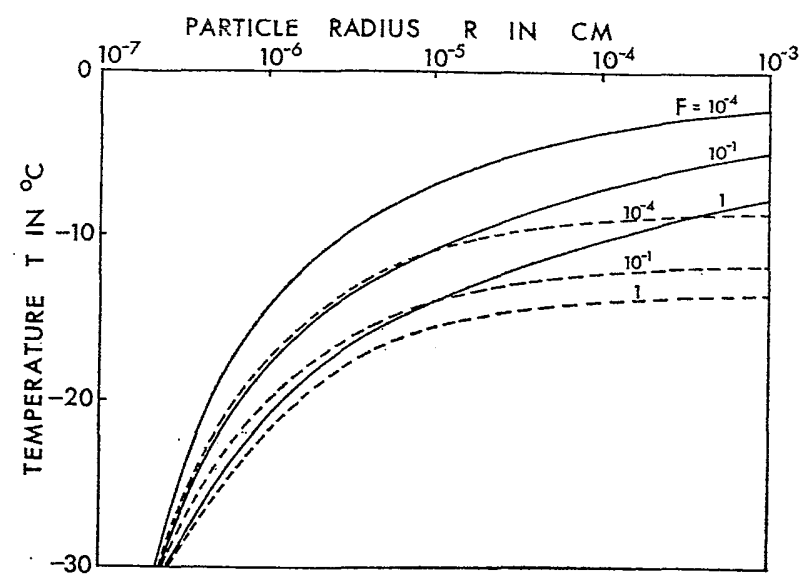

Frg. 4. Curves showing temperature $T$ above which a fraction $F$ of particles of radius $R$ are active as freezing nuclei. The full curves are for $\gamma=0.8, \beta=0.001$ and the broken curves for $\gamma=1.0$, $\beta=0.001$. The value of $F$ is shown as a parameter.

From Fig. 4, the set of curves with $\gamma=0.8$ seems to give a better approximation to experimental results than does the set for $\gamma=1.0$, so that it is worthwhile to generalize these to other values of $\beta$. From our earlier discussion of Fig. 3, the relevant parameter is really $F / \beta$ and Fig. 5 now presents generalized nucleation curves in terms of this parameter. Since there is some curvature in the lines of Fig. 3 near the knee, the curves of Fig. 5. are not completely accurate, but will suffice for our purpose.

If $\gamma$ does not vary significantly from one particle population to another, as seems reasonable, then each such population is characterized by its size distribution and by its value of $\beta$, which may vary widely in the range $\beta \ll 1$ depending upon the method of production. If these two characteristics are known, then Fig. 5 allows the nucleation behavior to be predicted. Con-

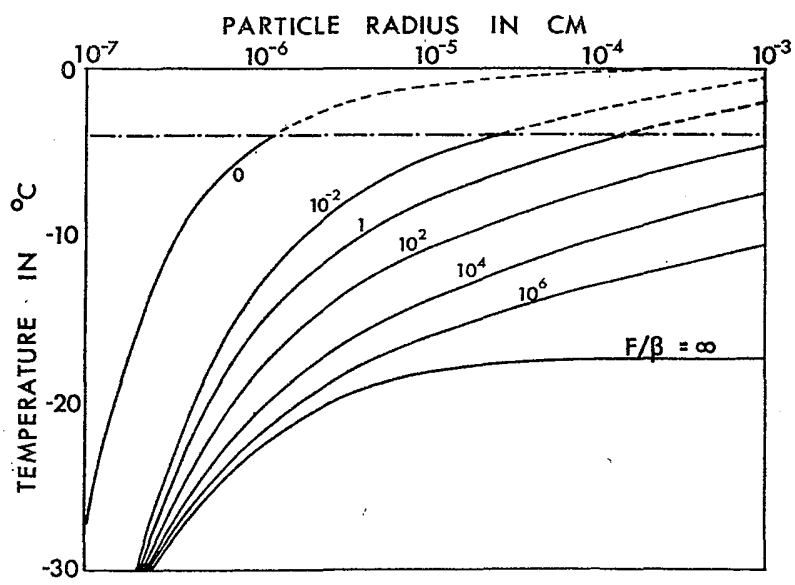

FIG. 5. Generalized approximate curves showing temperature $T$ above which a fraction $F$ of particles of radius $R$ and activity parameter $\beta$ act as freezing nuclei. $\gamma$ is taken to have the value 0.8 and the parameter shown is $F / \beta$. The region above $-4 \mathrm{C}$ is inaccessible. versely, if $\beta$ is not known, then Fig. 5 allows it to be determined from the observed nucleation behavior, provided our theory is applicable.

It is not the purpose of the present paper to attempt any detailed comparison between theory and experiment-the existence of the three parameters $m, \beta$ and $\gamma$ makes it clear that close agreement could be obtained using physically reasonable values-but rather to show that this simplest possible approach to the activesite problem is potentially able to account for the observed phenomena. It would now be a useful program to attempt further experimental investigations of these sites to see whether they have, in fact, the nature and distribution suggested here.

Acknowledgments. This work on ice nucleation is part of a program for study of the physics of disordered and statistical solids which is supported by the Australian Research Grants Committee.

\section{REFERENCES}

Abraham F. F., 1968: A re-examination of homogeneous nucleation theory: Thermodynamic aspects. J. Atmos. Sci., 25, 47-53.

Abramowitz, M., and I. A. Stegun, 1965: Handbook of Mathematical Functions. New York, Dover, Formula 7.1.13.

Edwards, G. R., L. F. Evans and V. K. La Mer, 1962: Ice nucleation by monodisperse silver iodide particles. $J$. Colloid $S c i$. 17, 749-758.

Edwards, L. F., and G. R. Evans, 1962 : Effect of surface charge on ice nucleation by silver iodide. Trans. Faraday Soc., 58, 1649-1655.

Federer, B., 1968: On the influence of the surface properties of semiconductors upon their ice nucleation ability. Z. Angew. Math. Phys., 19, 637-665.

Fletcher, N. H., 1958: Size effect in heterogeneous nucleation. J. Chem. Phys., 29, 572-576.

, 1959a: On ice-crystal production by aerosol particles. J. Meteor., 16, 173-180.

- $1959 \mathrm{~b}$ : The optimum performance of silver-iodide smoke generators. J. Meteor. ,16, 385-387.

- 1959 c: Entropy effect in ice crystal nucleation. J. Chem. Phys., 30, 1476-1482.

-_ 1960: Nucleation and growth of ice crystals upon crystalline substrates. Australian J. Phys., 13, 408-418.

- 1962: The Physics of Rainclouds. Cambridge University Press, Chap. 8.

- 1963 : Nucleation by crystalline particles. J. Chem. Phys., $38,237-240$.

- 1968 : Ice nucleation behavior of silver iodide smokes containing a soluble component. J. Atmos. Sci., 25, 1058-1060.

Katz, U., 1962: Cloud-chamber investigation of ice nucleation activity. Z. Angere. Math. Physics, 13, 333-358.

Mossop, S. C. and K. O. L. F. Jayaweera, 1969: AgI-NaI aerosols as ice nuclei. J. Appl. Meteor., 8, 241-248.

- , and C. Tuck-Lee, 1968: The composition and size distribution of aerosols produced by burning solutions of AgI and $\mathrm{NaI}$ in acetone. $J$. Appl. Meteor., 7, 234-240.

Turnbull, D., 1950: Kinetics of heterogeneous nucleation. $J$. Chem. Phys., 18, 198-203.

systems. $J$. Chem. Phys., 17, 71-73.

Zettlemoyer, A. C., N. Tcheurekdjian and C. L. Hosler, 1963: Ice nucleation by hydrophobic substrates. $Z$. Angew. Math. Physics, 14, 496-502. 